\title{
EVALUACIÓN DE LA CALIDAD EN EL SERVICIO Y SU IMPACTO EN LA LEALTAD EN BANCOMER
}

\section{ASSESMENT OF QUALITY IN SERVICE AND ITS IMPACT ON LOYALTY IN BANCOMER}

\section{Mauro Alejandro Monroy Ceseña (Universidad Autónoma De Baja California Sur) ${ }^{1}$}

\begin{abstract}
Resumen:
Este trabajo de investigación se realizó en 2 instituciones financieras BANCOMER en la ciudad de La Paz, Baja California Sur, México. El objetivo es determinar cómo inciden las medias de la calidad en el servicio en las medias de la lealtad del cliente en dos bancos de la misma firma empresarial, y encontrar si existe diferencia significativa entre todas las medias de las dimensiones que construyen la calidad en el servicio del cliente. Se realizó y validó un cuestionario basado en la metodología SERVPERF de Cronin y Taylor (1994) a través del coeficiente Alpha de Cronbach con un resultado de 0,83, además, se analizó, a la calidad en el servicio, y a la lealtad del cliente a través de una correlación de Pearson, obteniendo resultados de 0,85 . Por último, no fue posible encontrar diferencia significativa en las dimensiones de la calidad en el servicio percibido en las dos instituciones financieras BANCOMER.
\end{abstract}

Palabras clave: Calidad, servicios, lealtad, bancos.

Código JEL: M30; M31

\begin{abstract}
:
This research work was carried out in 2 financial institutions BANCOMER in the city of La Paz, Baja California Sur, Mexico. The objective is to determine how quality averages affect the service in the customer loyalty stockings in two banks of the same business firm, and find out if there is significant difference between the means of the dimensions that build the quality in Customer service. A questionnaire was conducted and validated based on the SERVPERF methodology of Cronin and Taylor (1994) through the Alpha coefficient of Cronbach with a result of. 0,85 , in addition; The quality of service and the loyalty of a Pearson correlation were analyzed, obtaining results from. 0,85. Finally, it was not possible to find a significant difference in the quality dimensions of the service perceived in the two BANCOMER financial institutions.
\end{abstract}

Key words: Quality, services, loyalty, Banks.

JEL Code: M30; M31

\footnotetext{
${ }^{1}$ monroym@uabcs.mx, Universidad Autónoma de Baja California Sur. Recibido 24 de febrero de 2019. Aceptado 11 de noviembre de 2019.
} 


\section{INTRODUCCIÓN}

El servicio al cliente constituye para las organizaciones una de las grandes exigencias que demanda una adecuada atención para lograr una impecable prestación de éste al momento de ser realizado. Debido a su relevancia, las empresas realizan un gran esfuerzo por entregar a sus clientes este conjunto de actividades que implican una naturaleza propia, con el fin de lograr que el usuario se sienta complacido y lo encuentre acorde con el valor invertido por su adquisición.

Sin embargo, existen factores que influyen en la entrega del servicio al cliente, que deben ser revisados para definir cómo intervenirlos, con el fin de mejorar esas acciones. Por esa razón, el presente trabajo de investigación se enfoca en uno de los aspectos más determinantes en ese resultado óptimo deseado, como lo es el estudio del servicio analizando el fenómeno de la calidad y el de la lealtad, debido a que esta genera una filosofía que caracteriza a la empresa e influye en la interacción interna del capital humano en la realización de sus esfuerzos individuales y grupales en el cumplimiento de sus labores.

Dentro del ámbito de la mercadotecnia, es común mencionar que la calidad es uno de los principales pilares de la mejora organizacional y partícipe de la construcción de un futuro direccionado, en gran medida, por las capacidades ejercidas para poder ofrecer productos y servicios a un mercado cada vez más demandante y competitivo. De ahí, el interés legítimo de los empresarios (tomadores de decisiones) por encontrar mecanismos o indicadores de valoración de la calidad de sus productos y servicios ofertados. Un servicio bancario desde el punto de vista de González (2001), es la actividad que desarrollan las entidades financieras como empresas de servicios; es decir, todas aquellas acciones que se establecen para servir a los clientes. Los servicios bancarios son complejos al combinar aspectos tangibles (pagos, retiros, depósitos y papelería) y aspectos intangibles (sensación de seguridad, capacidad de respuesta y responsabilidad de los funcionarios) (Cobra, 2002), y se caracterizan por sufrir rápidos cambios en su ambiente (Dávila y Florez, 2008), debido principalmente a la tecnología, y que, relacionados con la información, resultan ser cada vez más importantes.

Sin duda alguna, la calidad se ha presentado como una de las variables que todo cliente considera toral para su toma de decisiones con respecto a su consumo dentro de un mercado. Montgomery (2010), comenta que en el diseño de los productos y servicios es común que se preste poca atención a lo que opina el cliente y que el fenómeno de la calidad se perciba como el cumplimiento de las especificaciones sin importar si el producto es adecuado para su uso por parte del consumidor. Por otra parte, Ruiz (2006) menciona que dentro de los servicios se presenta una complejidad debido a las características inherentes al mismo. Aspectos como la intangibilidad, simultaneidad y heterogeneidad dificulta su control y la valoración de sus resultados, para lo cual se emplean métodos indirectos, a través de la percepción del cliente.

La calidad del servicio se define de manera general como una evaluación de la calidad desde la percepción de los clientes, donde se compara el servicio que esperan con las percepciones del servicio que ellos reciben, por lo que puede afirmarse que toda calidad es percibida por alguien (Cronin y Taylor, 1992). González Álvarez (2015) señala la importancia de centrarse en el cliente como único juez y evaluador de la calidad del servicio, cuyos criterios deben considerarse para garantizar la mejora continua de los procesos. Por otra parte, orientarse al cliente es un elemento crítico, puesto que este es activador en materia de servicio.

En consiguiente, en términos de lealtad a las empresas y dado el aumento de los niveles de competencia y competitividad en el contexto mundial, nacional y local, las instituciones financieras necesitan reconocer la importancia de atender correctamente las necesidades de sus clientes, con el propósito de alcanzar relaciones a largo plazo con él, y al mismo tiempo, lograr 
mantenerse vigentes en el mercado; es decir, se hace necesario identificar todas aquellas variables que influyen y determinan el comportamiento de los clientes.

Inicialmente existía la creencia de que, para crear lealtad era suficiente aumentar la satisfacción del cliente (Hallowell, 1996; Barlow, 1992), y con ese pensamiento bastaba para seguir activo en el mercado; sin embargo, los clientes no tienen ningún interés en ser leales a una empresa en particular. La lealtad del cliente es tan solo la respuesta a cómo el negocio presenta sus productos y servicios. Ahí está la clave, los clientes continuarán comprando a una empresa determinada, porque la relación que tienen con ella les parece atractiva y fructífera tanto en lo económico como en lo emocional.

Diversos estudios han comprobado que uno de los procesos principales para crear lealtad “consiste en atraer, mantener e intensificar las relaciones con los clientes” (Berry, 1995); dicho pensamiento, hace hincapié en alcanzar y mantener vínculos más allá de los comerciales existentes entre cliente y empresa, para así poder garantizar la lealtad de dichos consumidores.

En términos económicos, la lealtad, genera un impacto positivo, puesto que un cliente leal siempre volverá a adquirir un mismo producto, esto provoca una reducción en costos acerca de la captación de nuevos clientes. El costo de captación de nuevos clientes puede ser seis veces más que mantener un cliente actual (Bravo et al., 2005). Lo anteriormente comentado, se fortalece al analizar que, los clientes leales tienden a hacer un mayor gasto por un producto o servicio de lo presupuestado (Bender, 1976).

Con base en estos antecedentes, el propósito de esta investigación es evaluar la calidad en el servicio (CS) y su impacto hacia la lealtad del cliente (Lt) en las dos instituciones financieras BANCOMER en la ciudad de La Paz, México, a través del estudio de la percepción promedio de los clientes bajo múltiples dimensiones; de tal manera que, se identifiquen las dimensiones que impacten con efectos positivos o negativos, generando una primera línea base de indicadores de calidad. Otro objetivo importante en esta investigación es identificar si existe diferencia significativa en las medias de las dimensiones de la calidad en el servicio y en las medias de la lealtad del cliente en las dos instituciones financieras BANCOMER durante la realización del estudio; así mismo, con la información recabada se contribuirá tanto en la documentación académica como en la generación de un instrumento pragmático para la toma de decisiones.

\section{REVISIÓN DE LA LITERATURA}

\subsection{Calidad en el Servicio}

En relación con la medición de la Calidad de Servicio (CS), el instrumento más utilizado es el SERVQUAL (Parasuraman et al., 1985; 1988). Este instrumento evalúa la percepción en la CS con escalas multi-ítems en multi-dimensiones. La percepción de la calidad es el juicio de los clientes acerca de la excelencia o superioridad general de una entidad, como una forma de actitud relacionada pero no equivalente a la satisfacción (Parasuraman et al., 1988). De acuerdo con Oliver (1997), la satisfacción es la suma del estado psicológico que resulta cuando la emoción que rodea las expectativas no confirmadas se combinan con los sentimientos previos del consumidor sobre la experiencia del consumo. El mismo autor resume la naturaleza de la satisfacción como una transacción específica, por lo cual, una actitud es una orientación afectiva duradera de los consumidores hacia un producto o proceso (servicio al cliente), mientras que la satisfacción es una reacción emocional después de una experiencia no confirmada que actúa en el nivel de actitud base y es específica del consumo. En este sentido, tal como lo mencionan Parasuraman et al. (1988), la percepción de la CS es un juicio global o actitud, donde la satisfacción está relacionada con una transacción especifica. Bitner (1990), 
sustenta la importancia del modelo SERVQUAL debido al término disconformidad positiva/negativa, en el que las expectativas y las percepciones no son similares, lo anterior, lleva a suponer que, el modelo manifiesta que la satisfacción del cliente (SC) es un input en la percepción del cliente, reflejado en el comportamiento de compra. Rust y Oliver (1994), afirman que, los juicios posteriores de satisfacción son el resultado de la diferencia percibida por el consumidor entre sus expectativas y la percepción del resultado.

El diseño SERVQUAL ha sido ampliamente utilizado como base para el diseño de instrumentos de medición de la CS (Seth et al., 2005). El instrumento valora la CS en diez dimensiones de 97 ítems basado en una escala de Likert, donde en cada atributo de la CS se contemplan dos reactivos: uno sobre expectativas con respecto al atributo y otro, sobre el desempeño de éste, donde la diferencia (gap o brecha) se refiere a la CS. No obstante, Cronin y Taylor (1992), proponen una adaptación llamada SERVPERF (Service Performance) que mide únicamente el desempeño, demostrando ser una forma válida de medir la CS (Chinomona et al., 2013).

Jain y Gupta (2004), mencionan que el modelo SERVPERF tiene la ventaja de que sólo se aplica una vez, a diferencia del modelo SERVQUAL, tomando como principal resultado, no la diferencia entre las expectativas y las percepciones; sino que, únicamente considera a las percepciones, haciendo una operatividad mucho más sencilla en su aplicación. Si bien el instrumento SERVPERF presenta ventajas logísticas en la implementación de encuestas, la utilización de forma genérica en las diferentes industrias resultaría limitada, debido a que, la calidad como proceso depende en gran medida de dos aspectos: el tipo de industria y el contexto cultural, de acuerdo con (Guzmán López \& Cárcamo Solís , 2014). En este sentido, Bolton y Drew (1994), Heung, Wong, y Qu (2000), comentan que, cada industria posee sus particularidades, así mismo, las características de los clientes cambian de una industria a otra; por consiguiente, cada industria está conformada por diferentes dimensiones que le dan forma a la CS y llegan a requerir adiciones conforme se especifique en mayor medida el servicio de cada sector (Dabholkar et al., 1996). Las empresas dedicadas a los servicios tienen que ir no sólo en constante crecimiento y adaptabilidad conforme a las exigencias de los consumidores; sino que, también, éstas deben comprender que los competidores están cada vez más preparados y capacitados para ofrecer sus servicios. La medición de la CS, no únicamente se limita a los instrumentos como SERVQUAL o SERVPERF (Ladhari, 2008; Ramezani, Feiz, y Baharun, 2015), Service Quality model (Grönroos, 1984), SERVQUAL NQ y EP (Teas, 1993); debido a que existen otros instrumentos considerando las características de cada industria.

Prakash y Mohanty (2013), aseguran que la calidad de los servicios genera buenos dividendos cuando se hace bien, por lo que, invertir dinero y tiempo en su mejoramiento, aumenta las ventas, retiene al cliente y logra la exclusividad de uso por parte de los usuarios. Desde el punto de vista de Zeithaml y Bitner (2000), la calidad de servicio también se define como la percepción que tiene el cliente sobre el servicio recibido; dicha percepción, es producto de la discrepancia entre las expectativas que tenía antes de recibir el servicio y el desempeño actual. No obstante, los clientes no perciben la calidad de servicio como un concepto unidimensional, es decir, se hace una diferenciación de la información en varias dimensiones.

La calidad ha tomado un gran impulso, buscando no solo la satisfacción de las necesidades del cliente, sino que se perfila como un factor competitivo (Vargas \& Aldana, 2014). La evaluación de la satisfacción de los clientes debe ser un objetivo primordial en cualquier organización de servicio y el desarrollo de indicadores de la calidad es una forma adecuada para diagnosticar el desempeño de un proceso (Gutiérrez et al., 2014). Aunque la calidad del servicio es un proceso difícil de medir dado que, es subjetiva, han existido varias corrientes y aproximaciones sobre el tema para su mayor facilidad y comprensión, esto, debido 
a la creciente importancia que tienen los servicios en el sistema económico (Duque Oliva y Canas Baquero, 2014).

Una empresa dirigida al cliente debe preocuparse por cumplir con las expectativas que tienen los usuarios acerca de la calidad en el servicio que recibirán, además en el mundo de los negocios la competencia es muy fuerte, por lo que, las empresas que no le tomen importancia a medir la calidad en el servicio prestado, se encuentran bajo un enorme riesgo de ceder clientes a los negocios que ofrezcan servicios sustitutos. A veces, la única razón por la que un cliente toma su decisión de consumo entre dos o varios negocios que otorgan el mismo producto o servicio, es por la manera en que se siente atendido, ahí se presenta la diferencia entre un negocio exitoso y otro que, no lo es. Por lo general, realizar mejoras en la calidad del servicio no tiene un valor económico para las organizaciones, pero el cliente lo percibe (Hayes, 2013) y se fidelizará con la empresa (Zeithaml, 2000).

Las mejoras de la calidad del servicio dentro de una empresa no son trabajo exclusivo de la dirección, sino que, son todos los miembros de la organización los que deben participar en ello para que realmente surjan efectos positivos. El servicio al cliente constituye un factor intangible de la mercadotecnia de gran importancia para la apreciación de los clientes, por lo que la satisfacción se va diseñando a través de percepciones positivas continuas, alcanzando una lealtad hacia la empresa o hacia la marca.

Todos los consumidores tienen un concepto sobre qué es la calidad, pero difícilmente lo pueden definir; por lo general, ellos asumen que, un producto o servicio es de calidad cuando cumple con sus especificaciones o funciones para lo cual fue creado. Esto explica el porqué de la multidimensionalidad de la calidad, es decir, abarca distintas acepciones, dependiendo de quién lo describa; y esto, involucra necesariamente un juicio de valor de los individuos. Medir la calidad en el servicio es importante en la medida en que las empresas serán capaces de tomar las acciones o correctivos necesarios para mejorar su servicio ofertado al mercado; esto, a su vez mejorará la imagen de la compañía, lo cual, puede ir acompañado de un beneficio económico al aumentar las ventas, y lo más importante, otorgará una mejor experiencia de consumo/compra para los clientes.

\subsection{Calidad en el servicio en bancos}

El sector bancario no está ajeno a la evaluación constante del servicio, lo anterior, se debe al gran número de competidores existentes en el mercado. Sangeetha y Mahalingam (2011), identifican varios modelos aplicados al sector bancario con sus debidas fortalezas y limitaciones. A continuación, se analiza una serie de modelos, identificando las variables consideradas y las principales observaciones de los mismos.

\section{Modelo de Mersha y Adlakha}

El modelo propuesto por Mersha y Adlakha (1992), tiene como objetivo de estudio, identificar y clasificar los atributos de calidad desde la perspectiva de los consumidores (los autores modificaron y extendieron el instrumento SERVQUAL incluyendo preguntas adicionales para evaluar el núcleo del servicio, la personalización del servicio, el conocimiento e información). Para el servicio de la banca minorista, las características más importantes de buena calidad encontradas fueron: 1. Voluntad para corregir errores; 2. Exhaustividad / precisión; 3. Consistencia / fiabilidad; y, 4. Habilidad de conocimiento. Se encontró también que, las características más importantes de una mala calidad de servicio son: la renuencia a corregir errores; la indiferencia del empleado; falta de conocimiento sobre el servicio; $y$, un servicio descuidado o poco riguroso. 


\section{Modelo de Ennew, Reed y Binks}

En el análisis de la Importancia-Desempeño y la calidad del servicio de Ennew, Reed, y Binks (1993), los autores examinan los problemas relacionados con la medición de la calidad en el servicio para proporcionar medidas de expectativas, percepciones y la satisfacción general. Las 11 dimensiones de la calidad de los servicios utilizados en este análisis fueron: 1 . Conocimiento del negocio; 2. Conocimiento de la industria; 3. Conocimiento del mercado; 4. Otorgamiento de consejos útiles; 5. Amplitud en la gama de servicios; 6. Tasas de interés competitivas; 7. Cargos/Costos competitivos; 8. Rapidez de la decisión; 9. Diseño de las finanzas; 10. Negociar con una persona; y 11. Fácil acceso a oficial de préstamo.

\section{Modelo de Avkiran}

La escala de la calidad del servicio al cliente propuesto por Avkiran (1994), tuvo como propósito desarrollar un instrumento multidimensional que, pudiera ser aplicado para la medición de la calidad del servicio al cliente según su percepción de la red de sucursales bancarias. Los autores tomaron como punto de partida el trabajo desarrollado por Parasuraman et al. (1985). El trabajo resultó en un instrumento de 17 puntos en 4 dimensiones para medir la calidad del servicio al cliente en la red de sucursales bancarias, con un enfoque en la banca minorista. Las cuatro dimensiones que surgieron son: 1. Conducta del personal; 2. Credibilidad; 3. Comunicación; y, 4. Acceso a los servicios de cajero.

\section{Modelo de Blanchard y Galloway}

El modelo de calidad del servicio de Blanchard y Galloway (1994), determina la percepción de los clientes con respecto a los requerimientos de calidad en el servicio en el negocio de los bancos utilizando el modelo SERVQUAL (1988). Esto, se debe a que, dicho modelo no fue exitoso para proporcionar alguna idea acerca de cómo mejorar el servicio. Este modelo propuesto se basa en tres dimensiones: el proceso - resultado (diseño de servicios y resultados); subjetivo - objetivo (medida del grado en que la calidad en el servicio puede ser objetivamente valorada) y “soft - hard” (aspectos físicos del servicio y la interacción personal).

\section{Modelo de Johnston}

El modelo de los factores de calidad del servicio basado en la satisfacción propuesto por Johnston (1997), clasifica los factores de calidad en términos de su importancia relativa y su efecto sobre la satisfacción y la insatisfacción. Los 18 factores determinantes de la calidad de los servicios propuestos son: 1. Accesibilidad; 2. Estética; 3. Atención; 4. Disponibilidad; 5. Cuidado; 6. Limpieza/Pulcritud; 7. Confort; 8. Compromiso; 9. Comunicación; 10. Competencia; 11. Cortesía; 12. Flexibilidad; 13. Amabilidad; 14. Funcionalidad; 15. Integridad; 16. Fiabilidad; 17. Capacidad de respuesta; y 18. Seguridad.

\section{Modelo de Joseph, Mcclure y Joseph}

El modelo desarrollado por estos autores está basado en la tecnología utilizada para la entrega de la calidad del servicio en donde, el uso de esta tecnología en la prestación de servicios realizada por los bancos es cada vez más frecuente ya que permite reducir los costos y eliminar incertidumbres. Este estudio se centra en el papel que desempeña la tecnología en la banca australiana y su impacto en la prestación de la calidad del servicio percibida.

Joseph et al. (1999), utilizaron la cuadrícula de Hemmasi, Strong, y Taylor (1994), e identificaron un modelo de seis factores consistentes en: 1. Conveniencia / precisión; 2. 
Retroalimentación / Gestión de reclamaciones; 3. Eficiencia; 4. Gestión de colas; 5. Accesibilidad; y 6. Personalización.

\section{Modelo de Bahía y Nantel}

En el modelo para la calidad del servicio bancario de Bahia y Nantel (2000), los autores sostienen que los instrumentos disponibles no incluyen una escala diseñada para bancos específicos o instrumentos diseñados específicamente para el servicio bancario; sino que, su diseño se basa únicamente para una amplia gama de servicios. Dado lo anterior, los autores proponen una escala de 31 puntos para medir la calidad percibida de los servicios bancarios que abarcan seis dimensiones: 1. Efectividad y seguridad; 2. Accesibilidad; 3. Precio; 4. Tangibles; 5. Portafolio de servicios; y 6. Fiabilidad.

\section{Modelo de Sureshchandar, Rajendran y Kamalanabhan}

Sureshchandar et al. (2001), revisaron exhaustivamente el modelo de SERVQUAL (1988) y sus 22 puntos, lo que implicó analizarlos desde la interacción/intervención de las personas en la entrega del servicio, además de las facetas tangibles en un proceso de servicio. El instrumento parece haber pasado por alto algunos otros factores importantes, por ejemplo: Servicio principal (producto), sistematización / estandarización de la prestación de servicios (el elemento no humano) y la responsabilidad social de la organización. En un esfuerzo por conceptualizar la calidad del servicio, estos autores identificaron cinco factores de calidad de servicio que son: 1 . Servicio principal producto.; 2. Elemento Humano en la prestación del servicio; 3. Sistematización de la prestación del servicio; 4. Tangibles; y 5. Responsabilidad social de la organización.

\section{Modelo de Aldlaigan y Buttle}

El Modelo SYSTRA propuesto por Aldlaigan y Buttle (2002), sostiene a un instrumento para medir la calidad percibida basado en el modelo de Grönroos (1984), realizado especialmente para los servicios bancarios, concluyendo en una escala de 21 ítems basados en la calidad a nivel organizacional y transaccional. Desarrolló las siguientes 4 dimensiones: 1. Calidad del sistema de servicio; 2. Calidad de comportamiento del servicio; 3. Calidad de servicio de las máquinas; y, 4. Transacción del servicio.

\section{Modelo de Al-Hawari, Hartley y Ward}

Para Al-Hawari et al. (2005), la calidad del servicio automatizado se plantea como el factor que determina el éxito o el fracaso del comercio electrónico. Los autores afirman que los modelos disponibles en la actualidad están limitados en su enfoque, porque abarcan sólo un canal electrónico (Internet), haciendo caso omiso de los demás canales de servicio automatizados. En relación con el sector bancario, las investigaciones han identificado que los clientes tienden a utilizar una combinación de canales. Por lo tanto, los autores se esfuerzan por desarrollar un modelo integral teniendo en cuenta las características únicas de cada canal de distribución y otras dimensiones que tienen una influencia potencial sobre la calidad. Los autores proponen cinco factores: 1 . Servicio de cajeros automáticos; 2 . Servicio de banco por Internet; 3. Servicio de banco por teléfono; 4. Servicio básico; y 5. Percepción del cliente sobre el precio. 


\section{Modelo de Karatepe, Yavas y Babakus}

La escala de calidad de servicio para la banca propuesta por Karatepe et al. (2005), se desarrolla como un instrumento de 20 puntos para medir las percepciones de los clientes sobre la calidad del servicio bancario en el norte de Chipre. Los resultados mostraron que la calidad del servicio podría ser conceptualizada y medida a través de cuatro dimensiones: 1 . Ambiente del servicio; 2. Calidad de la interacción; 3. Empatía; y, 4. Confiabilidad.

\section{Modelo de Ehigie}

El modelo de las expectativas y servicio percibido por parte de los clientes de Ehigie (2006), en el que se desarrolla una escala mediante la técnica cualitativa para medir las variables que intervienen en las expectativas del cliente, la calidad de servicio percibida y la satisfacción que impactan en la lealtad de los clientes hacia los bancos en Nigeria, originó las siguientes variables: 1 . trabajadores del banco con el dominio de las habilidades requeridas; 2. trabajadores del banco con el conocimiento y experiencia requerida; 3. continuidad del servicio al cliente en los próximos años; 4. entender las necesidades de los clientes; 5 . oferta de un servicio rápido y eficiente; 6 . proporcionar seguridad física a los clientes; 7. confidencialidad de las transacciones; 8 . actitud positiva del personal de servicios al cliente; 9. fiabilidad de banco; 10. buena reputación del banco; 11. amabilidad del personal; 12. mantener a los clientes informados, 13. escuchar a los clientes; 14. la inclusión de servicio bancario los sábados; 15. horarios bancarios extendidos; y, 16. la cobertura del seguro para los clientes.

\subsection{Lealtad en el cliente}

En las últimas décadas se han presentado cambios dentro del ambiente competitivo, creando la necesidad de una perspectiva que va desde un mercado global hasta una relación individual con el cliente. De acuerdo con este enfoque, en donde la prioridad es establecer una relación cercana y reciproca con los consumidores, los programas orientados hacia la lealtad se han convertido en una herramienta estratégica de gran relevancia para la retención de los clientes (Pedraja y Rivera, 2002).

Dávila Nima (2007), se refiere a la lealtad como el objetivo último del nuevo marketing para obtener empresas líderes; es decir, en el marketing actual, el objetivo final es el de establecer relación con el cliente desde su primera compra, creando un lazo de confianza entre el cliente y la empresa, de tal forma que la competencia se vea limitada.

Shamsudin et al. (2016) aseveran que, para el éxito a largo plazo de una empresa, será un factor predominante mantener clientes leales; es decir, se hace necesario tener en cuenta que en el entorno actual existe un mercado altamente competitivo, lo que hace a la lealtad del cliente un factor de mayor importancia.

De acuerdo con los académicos, los clientes que se consideran habituales (aquellos que suelen repetir sus experiencias en un mismo servicio) son los responsables de generar mayores rendimientos para las empresas, por lo que, es posible afirmar que, mantener o rentabilizar la relación con un cliente conocido es más rentable que captar un cliente nuevo; pero en la realidad, en los mercados emergentes, a pesar de esta realidad, cada vez resulta más difícil mantener la lealtad de los clientes, y esto se debe a eso que los gerentes de las organizaciones empiezan a cuestionarse ¿por qué los clientes abandonan el servicio? Es importante empezar por entender qué significa lealtad y los tipos de ésta de acuerdo con la rentabilidad que éstos representan para las organizaciones (Lovelock et al., 2004).

La lealtad ha sido considerada como algo más que una larga relación de un cliente con una marca determinada o la frecuencia con la que un cliente visita o compra en una tienda, es 
más bien, la sensación de afinidad que los clientes sienten con los productos o servicios de esta marca (Kumar y Shah, 2004). Se reconocen distintos tipos de lealtad: (a) la primera fidelidad, que se produce cuando existen una correspondencia favorable entre la actitud del individuo frente a la empresa y su comportamiento de compra; ésta, representa la situación ideal para la compañía y para el cliente, en estos casos, generalmente los clientes suelen actuar como embajadores de la marca; (b) fidelidad latente, el cliente tiene una actitud positiva frente a la marca, pero no manifiesta una lealtad realmente significativa. Conocidos como clientes mercenarios, suelen ser clientes que tienen opiniones favorables de la empresa pero no suelen tener comportamiento estable de compra por factores externos, generalmente económicos; (c) fidelidad espuria, en este tipo el cliente presenta un alto comportamiento de compra, pero generalmente tiene malas opiniones de la marca, generalmente es un cliente rehén, es decir que no tiene la opción de cambiar de proveedor de su servicio; (d) No fidelidad, en este caso, no existe ni actitud ni comportamiento de compra del cliente hacia la marca, llamados clientes terroristas, suelen darle mala publicidad a la marca (Dick y Basu, 1994).

Dentro de los estudios del comportamiento del consumidor, se han considerado diferentes factores que implican cambios entre la selección del consumidor de una u otra marca; entre estos factores, se encuentra la lealtad. Su importancia en el comportamiento del consumidor ha generado un alto interés en el campo de la mercadotecnia (Jensen y Hansen, 2006).

Harris y Goode (2004), aseveran que los clientes leales son menos vulnerables al precio, actúan como canales publicitarios y efectúan mayores compras; por consiguiente, los costos e ingresos de una organización no se verán afectados y es aquí donde radica la importancia de obtener clientes leales en una organización. El mantener clientes actuales es menos costoso que el hecho de incorporar nuevos clientes, especialmente, en los mercados competitivos (Ehrenberg y Goodhardt, 2002).

Desde el punto de vista de la rentabilidad, diferentes estudios han demostrado que, mantener clientes leales genera los siguientes beneficios: los clientes leales realizan mayores compras y tienden con mayor frecuencia a repetir la compra, adquieren más otros productos de la misma empresa, su costo de operación es menor, realizan un "boca a boca” positivo, lo que aumenta la captación de clientes nuevos y son considerados por la empresa como una fuente importante para el desarrollo de nuevos negocios (Soriano, 2002). Asimismo, se ha demostrado que las compañías que poseen un alto porcentaje de clientes leales tienen una alta participación en el mercado (Jensen y\& Hansen, 2006).

\section{METODOLOGÍA}

La presente investigación posee un enfoque cuantitativo, ya que se realizó la recolección de datos con base en la medición numérica y el análisis estadístico de los mismos. Para conocer con mayor detalle el contexto de servicios financieros de BANCOMER en la ciudad de La Paz, fue necesario también utilizar la entrevista personal a autoridades de BANCOMER, siendo este un instrumento de corte cualitativo.

Por su alcance, este es un trabajo descriptivo-correlacional (Hernández et al., 2014), misma que se realizó con la finalidad de establecer una descripción lo más completa posible de un fenómeno y establecer la relación dada entre las variables de calidad en el servicio como variable independiente y de la lealtad del cliente como variable dependiente.

La manipulación de las variables se dio de forma no experimental, puesto que se recopilaron datos para observar el comportamiento de la calidad en el servicio en el contexto natural. El diseño de esta investigación es Transeccional o Transversal debido a que se recolectaron los datos desde el 26 de agosto al 23 noviembre de 2018 en las dos instituciones financieras. 
Como sujetos de estudio de esta investigación se tomaron a los clientes de las dos instituciones financieras BANCOMER en la ciudad de La Paz, México, aplicando cuestionarios previamente validados para conocer sus apreciaciones respecto a la calidad en el servicio bancario percibido y el nivel de lealtad generado por el servicio que se recibió.

Una vez diseñada la encuesta y las escalas de valoración para la CS y Lt, el siguiente paso fue explorar la consistencia interna de la encuesta (Hernández et al., 2013; Hernández et al., 2014). Por consiguiente, se realizó un muestro piloto de 10 encuestas (tres por especialidad) para establecer la consistencia interna mediante la estimación del alfa de Cronbach (Bonnet, 2002):

$$
\alpha=\left[\frac{k}{k-1}\right]\left[1-\frac{\sum_{i=1}^{k} s_{i}^{2}}{s_{t}^{2}}\right]
$$

Donde $\alpha=$ alfa de Cronbach, $k=$ número de ítems, $s_{i}^{2}=$ varianza del ítem $i, s_{t}^{2}=$ varianza de la suma de todos los ítems. Este estadístico, se utiliza para evidenciar la validez y confiabilidad de las encuestas en estudios basados, por ejemplo, en la escala de Likert. Un alfa de Cronbach se valora entre 0 y 1 , donde los valores por arriba de 0.7 (o 70 por cien) se interpreta como un instrumento de medición aceptable (Hernández et al., 2014). No obstante, en este estudio se realizó una estimación basada en la inferencia del alfa se Cronbach con intervalos de confianza, que de acuerdo con Domínguez y Merino (2015), es poco utilizado en los estudios en ciencias de la humanidad, pero la estimación permite garantizar estadísticamente la validez del instumento utilizado y sobre todo, la validación de la adaptación en los ítems respecto a la SC. Por lo cual se utilizó un intervalo de confianza del 95 por cien mediante el soporte estadístico IBM SPSS Statistics versión 23 de acuerdo con Bonnet (2002).

Los resultados que se pudieron encontrar una vez hecha la prueba piloto de 10 encuestas fue de 0,85, lo que prueba ser un instrumento confiable desde el punto de vista de Domínguez y Merino, (2015). De forma particularizada, se muestran los siguientes resultados en el cuadro 1:

CUADRO 1. ALPHA DE CRONBACH POR DIMENSIÓN DE LA CS

\begin{tabular}{|l|c|}
\hline \multicolumn{1}{|c|}{ DIMENSIONES } & $\infty$ \\
\hline TANGIBILIDAD & 0,8837 \\
\hline FIABILIDAD & 0,8072 \\
\hline CAP. RESPUESTA & 0,8607 \\
\hline SEGURIDAD & 0,8786 \\
\hline EMPATÍA & 0,8143 \\
\hline
\end{tabular}

Fuente: Elaboración propia. 


\subsection{Diseño del instrumento de investigación}

El instrumento de medición de la calidad en el servicio utilizado inicialmente fue una adaptación del modelo cuantitativo multidimensional denominado SERVPERF (cuadro 2) desarrollado por Cronin y Taylor (1994), el cual fue diseñado por los autores basándose en las mismas 5 dimensiones propuestas en el modelo SERVQUAL (cuadro 2) pero con un enfoque especifico a BANCOMER.

\begin{tabular}{|c|c|}
\hline \multicolumn{2}{|c|}{ CUADRO 2. DEFINICIÓN DE LAS DIMENSIONES DE LA CS } \\
\hline \multicolumn{2}{|r|}{ Modelo SERVPERF } \\
\hline Dimensión & Definición \\
\hline 1. Aspectos tangibles & $\begin{array}{l}\text { Relacionados con la apariencia de las instalaciones } \\
\text { físicas, equipo, personal, y material de comunicación. } \\
\text { Son los aspectos físicos que el cliente percibe, tales } \\
\text { como el diseño físico del restaurante, la apariencia del } \\
\text { personal de contacto y la limpieza del lugar. }\end{array}$ \\
\hline 2. Confiabilidad & $\begin{array}{l}\text { Implica cuestiones relacionadas con la fiabilidad de los } \\
\text { productos y servicios financieros percibidos por el } \\
\text { cliente. }\end{array}$ \\
\hline $\begin{array}{l}\text { 3. Respuesta (ante las } \\
\text { demandas del cliente) }\end{array}$ & $\begin{array}{l}\text { Referente a la asistencia personal del banco o la } \\
\text { respuesta adecuada y la rápida asistencia respecto a las } \\
\text { necesidades y peticiones de los clientes. }\end{array}$ \\
\hline $\begin{array}{l}\text { 4. Garantías } \\
\text { (aseguramiento o } \\
\text { cumplimiento) }\end{array}$ & $\begin{array}{l}\text { Entendido como que los clientes del banco deben ser } \\
\text { capaces de confiar en las recomendaciones del personal } \\
\text { de contacto, tener la seguridad de que los productos y } \\
\text { servicios financieros proporcionen seguridad, así como } \\
\text { poder expresar alguna preocupación, inquietud o } \\
\text { pregunta sin temor alguno. }\end{array}$ \\
\hline 5. Empatía & $\begin{array}{l}\text { Concerniente a la capacidad de brindar una atención } \\
\text { personalizada a los clientes mediante la previsión de sus } \\
\text { necesidades o por la disposición del personal de ser } \\
\text { comprensivo hacia las necesidades de los clientes. }\end{array}$ \\
\hline
\end{tabular}


La adecuación de los modelos mencionados permitió realizar un análisis estadístico para establecer patrones de comportamiento de los clientes, identificando esencialmente la percepción sobre la calidad del servicio percibido y el nivel de lealtad asimilado por los clientes. La versión final del instrumento de investigación compone entonces de 22 ítems acerca de la CS y cinco ítems relacionados a la Lt: La forma de medición de cada uno de los ítems del cuestionario fue a través de una escala de Likert de 7 puntos (cuadro 3).

\section{CUADRO 3. ESCALA DE LIKERT Y PUNTUACIONES UTILIZADAS EN EL INSTRUMENTO DE MEDICIÓN.}

\begin{tabular}{|c|c|}
\hline \multicolumn{2}{|c|}{ Escala de Likert } \\
\hline Puntaje & Juicio \\
\hline 7 & Totalmente de acuerdo \\
\hline 6 & Muy de acuerdo \\
\hline 5 & De acuerdo \\
\hline 4 & Ni de acuerdo ni en desacuerdo \\
\hline 3 & En desacuerdo \\
\hline 2 & Muy en desacuerdo \\
\hline 1 & Totalmente en desacuerdo \\
\hline
\end{tabular}

Fuente: elaboración propia. 
El instrumento de investigación que se aplicó a los clientes de las dos instituciones financieras BANCOMER en la ciudad de La Paz, México incluye las variables CS y Lt, y quedan constituidas tal y como se muestra en el cuadro 4:

\begin{tabular}{|c|c|}
\hline \multicolumn{2}{|r|}{ CUADRO 4. INSTRUMENTO DE INVESTIGACIÓN. } \\
\hline DIMENSIONES & REACTIVOS DE MEDIDA \\
\hline \multirow{5}{*}{ TANGIBILIDAD } & 1. BANCOMER tiene equipos de apariencia moderna. \\
\hline & 2. Sus instalaciones físicas son visualmente atractivas \\
\hline & 3. Sus empleados están bien vestidos y aseados \\
\hline & $\begin{array}{l}\text { 4. La apariencia de sus instalaciones se conserva de acuerdo al tipo de } \\
\text { servicio ofrecido }\end{array}$ \\
\hline & $\begin{array}{l}\text { 5. Contaré los aspectos positivos sobre la apariencia de BANCOMER } \\
\text { a otras personas }\end{array}$ \\
\hline \multirow{6}{*}{ FIABILIDAD } & 6. Cuando promete hacer algo en cierto tiempo realmente lo hace \\
\hline & $\begin{array}{l}\text { 7. Cuando usted tiene un problema, BANCOMER muestra interés en } \\
\text { solucionarlo }\end{array}$ \\
\hline & 8. BANCOMER es confiable en cada uno de sus procedimientos \\
\hline & $\begin{array}{l}\text { 9. BANCOMER provee sus servicios en el tiempo en que promete } \\
\text { hacerlo }\end{array}$ \\
\hline & 10. BANCOMER mantiene sus registros de manera correcta \\
\hline & $\begin{array}{l}\text { 11. Recomendaré a BANCOMER sobre su buen funcionamiento y } \\
\text { orientación al cliente (Intención de recomendación) }\end{array}$ \\
\hline \multirow{5}{*}{$\begin{array}{l}\text { CAPACIDAD DE } \\
\text { RESPUESTA }\end{array}$} & $\begin{array}{l}\text { 12. BANCOMER informa a los clientes exactamente cuando los } \\
\text { servicios serán ejecutados }\end{array}$ \\
\hline & 13. Usted recibe un servicio rápido de los empleados de BANCOMER \\
\hline & $\begin{array}{l}\text { 14. Los empleados de BANCOMER siempre están dispuestos a ayudar } \\
\text { a los clientes }\end{array}$ \\
\hline & $\begin{array}{l}\text { 15. Los empleados de BANCOMER están atentos a responder } \\
\text { rápidamente las peticiones de los clientes }\end{array}$ \\
\hline & $\begin{array}{l}\text { 16.Recomendaré consumir productos y servicios en BANCOMER } \\
\text { por la excelente respuesta recibida }\end{array}$ \\
\hline \multirow{5}{*}{ SEGURIDAD } & 17. Usted puede confiar en los empleados de BANCOMER \\
\hline & 18. Usted percibe seguridad en sus transacciones con los empleados \\
\hline & 19. Los empleados de BANCOMER son educados \\
\hline & $\begin{array}{l}\text { 20. Los empleados reciben apoyo adecuado de BANCOMER para } \\
\text { hacer bien su trabajo }\end{array}$ \\
\hline & $\begin{array}{l}\text { 21. Contaré sobre el excelente servicio mostrado por los empleados } \\
\text { de BANCOMER }\end{array}$ \\
\hline \multirow{6}{*}{ EMPATÍA } & 22. BANCOMER le da a usted una atención individualizada \\
\hline & $\begin{array}{l}\text { 23. Los empleados de BANCOMER le dan a usted una atención } \\
\text { personalizada }\end{array}$ \\
\hline & $\begin{array}{l}\text { 24. Los empleados de BANCOMER conocen cuáles son sus } \\
\text { necesidades }\end{array}$ \\
\hline & 25. BANCOMER considera sus intereses como objetivo \\
\hline & $\begin{array}{l}\text { 26. BANCOMER tiene horarios de operación convenientes para sus } \\
\text { clientes }\end{array}$ \\
\hline & $\begin{array}{l}\text { 27. Recomendaré a BANCOMER por la gran capacidad de } \\
\text { entenderme como cliente }\end{array}$ \\
\hline
\end{tabular}

Fuente: elaboración propia. 
Por otra parte, para determinar el tamaño de la muestra en este trabajo de investigación fue necesario y es importante mencionar que se platicó con las personas que están en ventanilla en BANCOMER, e informaron que durante una semana de trabajo altamente demandado el número de personas que asisten a realizar operaciones es alrededor de 400 . Considerando este dato como un valor poblacional $(\mathrm{N}=400)$, el tamaño muestral se calculó a través de la fórmula estadística de las poblaciones finitas considerando un error de estimación del 5 por cien, un nivel de confianza del 95 por cien, una probabilidad a favor $(p)$ del 50 por cien y una probabilidad en contra $(q)$ del 50 por cien, tal y como se muestra a continuación:

$$
\begin{aligned}
& n=(\sigma) 2(N) *(p) *(q) /(e) 2(N-1)+(\sigma) 2 *(p) *(q) \\
& n=(1.96) 2(400) *(.50) *(.50) /(.05) 2(400-1)+(1.96) 2 *(.50) *(.50) \\
& \mathrm{n}=196
\end{aligned}
$$

Lo anteriormente relatado, se puede visualizar con mayor comprensión en el cuadro 5 donde se presenta la ficha técnica en este trabajo de investigación:

\begin{tabular}{|l|c|}
\hline \multicolumn{2}{|c|}{ CUADRO 5. FICHA TÉCNICA DE LA INVESTIGACIÓN } \\
\hline OBJETIVOS & $\begin{array}{c}\text { Evaluar la Calidad en el Servicio y su } \\
\text { incidencia en la lealtad del cliente de la } \\
\text { institución financiera BBVA Bancomer }\end{array}$ \\
\hline UNIVERSO & 400 usuarios \\
\hline TAMAÑO MUESTRAL & Se establece un error del 5 por cien \\
\hline ERROR MUESTRAL & 95 por cien \\
\hline CONFIABILIDAD & Muestreo para poblaciones finitas \\
\hline TÉCNICA APLICADA EN CAMPO & Del 26 de agosto al 23 de noviembre de 2018 \\
\hline TRABAJO DE CAMPO & \\
\hline
\end{tabular}




\section{RESULTADOS}

Los resultados que se presentaron durante el trabajo de campo en la realización de esta investigación se pueden diseminar tanto a nivel descriptivo como a nivel inferencial, con el objetivo de responder las preguntas de investigación que se realizaron al inicio de este artículo.

A nivel descriptivo, la CS presentó un comportamiento positivo desde la perspectiva de los clientes asiduos a BANCOMER, en las instituciones financieras 1 y 2 donde se realizó la investigación concerniente al fenómeno de estudio. Los resultados se muestran tal y como se presentan en el cuadro 6 y en la figura 1:

\section{CUADRO 6. COMPORTAMIENTO DE LAS DIMENSIONES DE LA CS EN BANCOMER 1}

\begin{tabular}{|l|c|c|}
\hline DIMENSIONES & BANCOMER 1 & BANCOMER 2 \\
\hline TANGIBILIDAD & 5,71 & 5,70 \\
FIABILIDAD & 5,90 & 5,90 \\
CAP. RESP. & 5,82 & 5,82 \\
SEGURIDAD & 5,84 & 5,95 \\
EMPATÍA & 5,94 & 6,22 \\
\hline Media & $\mathbf{5 , 8 4}$ & $\mathbf{5 , 9 2}$ \\
\hline Efectividad & $\mathbf{8 3 , 4 7}$ por cien & $\mathbf{8 4 , 5 4}$ por cien \\
\hline
\end{tabular}

Fuente: Elaboración propia.

Como puede observarse, los resultados acerca de la CS en las dos instituciones financiera de BANCOMER presentaron una efectividad de servicio bastante aceptable por parte del cliente, ya que, éstos oscilaron entre el 82 por cien y el 85 por cien, con una media que representó el 84 por cien desde la perspectiva del cliente. Cabe agregar, que las dimensiones tangibilidad y empatía sobresalen en los resultados debido a un comportamiento por debajo de la media de servicio percibido (5,71 y 6,08 respectivamente). Lo anteriormente comentado es posible observarlo en la figura 1:

\begin{tabular}{|c|c|c|}
\hline FIGURA 1. COMPORTAMIENTO DE LAS DIMENSIONES DE LA CS EN \\
BANCOMER 1 Y 2 \\
\hline 6,25 \\
6,15 \\
6,05 \\
5,95 \\
5,85 \\
5,75
\end{tabular}


En lo que respecta a la lealtad del cliente (Lt), los resultados manifiestan similitud con respecto a lo observado a la opinión del cliente acerca del servicio percibido, ya que, al evaluar la efectividad del comportamiento hacia la lealtad del servicio adquirido en las dimensiones que se analizaron durante el estudio, éste, presentó una diferencia mínima en sus medias; es decir, en la institución BANCOMER 1 la media de lealtad fue de 83.46 por cien, mientras que la institución BANCOMER 2 fue sólo de 83.87 por cien, lo que representa una diferencia de .50 por cien entre una institución y otra en la localidad; sin embargo, hay tres factores que pueden llamar la atención debido a sus resultados por debajo de la media que se generó en el estudio, éstos son: tangibilidad, capacidad de respuesta y seguridad. Aun así, a pesar de que los resultados son aceptables desde la perspectiva de la efectividad calculada hacia la lealtad del cliente, éstos, presentan una diferencia significativa en relación con los otros dos factores considerados en el estudio (fiabilidad y empatía). Lo anterior, se puede observar en el cuadro $7 \mathrm{y}$ en la figura 2:

\section{CUADRO 7. COMPORTAMIENTO DE LAS DIMENSIONES DE LA LT EN BANCOMER 1 Y 2}

\begin{tabular}{|l|c|c|}
\hline DIMENSIONES & BANCOMER 1 & BANCOMER 2 \\
\hline TANGIBILIDAD & 5,80 & 5,78 \\
FIABILIDAD & 5,84 & 5,96 \\
CAP. RESP. & 5,89 & 5,82 \\
SEGURIDAD & 5,84 & 5,81 \\
EMPATÍA & 5,85 & 5,99 \\
\hline Media & $\mathbf{5 , 8 4}$ & $\mathbf{5 , 8 7}$ \\
\hline Efectividad & $\mathbf{8 3 , 4 6}$ por cien & $\mathbf{8 3 8 7}$ por cien \\
\hline
\end{tabular}

Fuente: Elaboración propia.

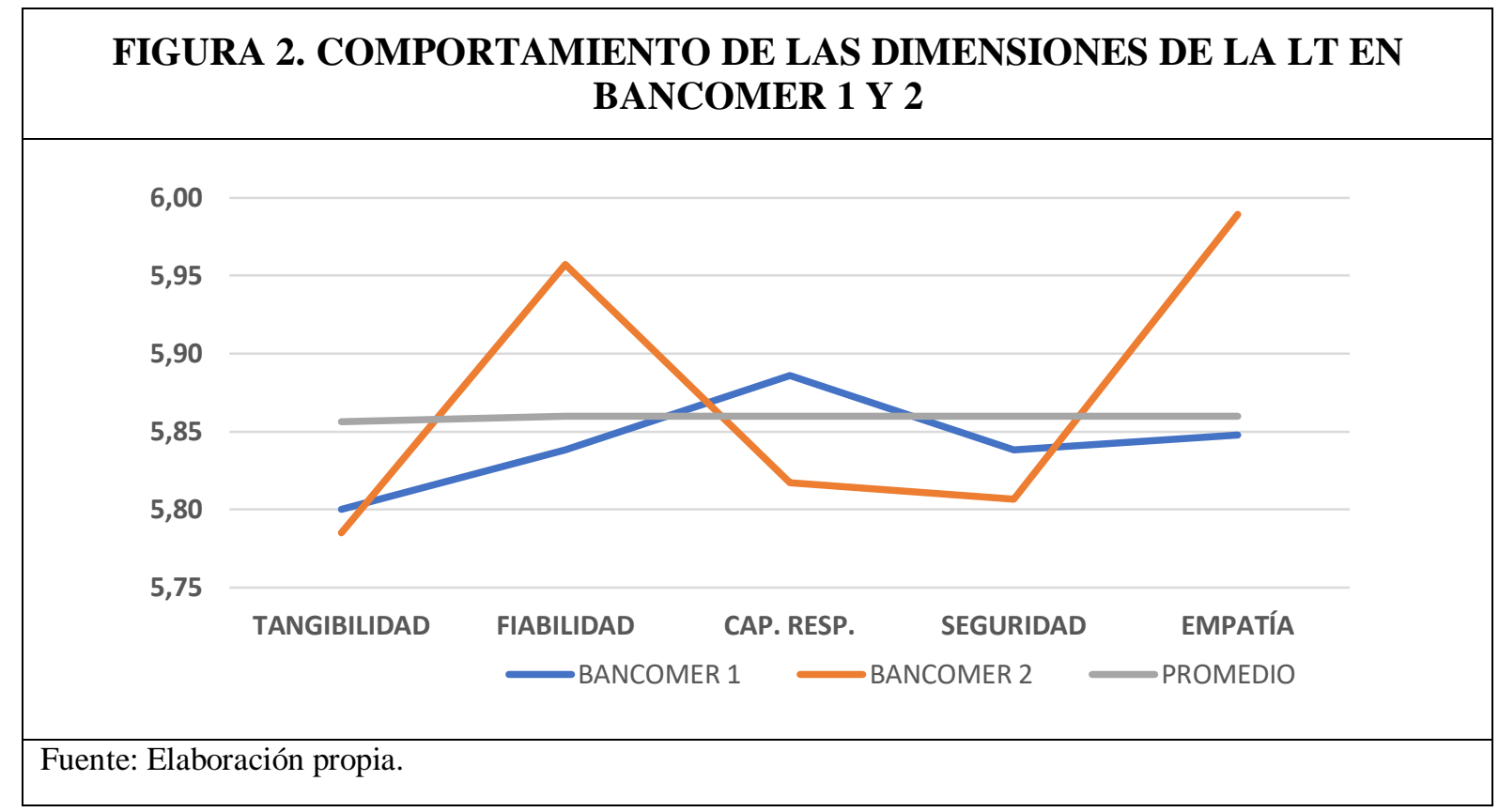


En lo que corresponde a la pregunta de investigación \#1, donde se buscó evaluar la calidad en el servicio (CS) y su impacto hacia la lealtad del cliente (Lt) en las dos instituciones financieras BANCOMER en la ciudad de La Paz, México, es posible observar como el comportamiento de los datos de las medias CS y de la Lt presentan una alta correlación entre ambos constructos, ya que al calcular el coeficiente de correlación de Pearson se obtuvo una calificación de 0,85 con un $\mathrm{R}^{2}$ de 0,72 , lo que desde el punto de vista de Taucher (1997), existe una buena determinación de la variable independiente (CS) sobre la variable dependiente (Lt). Lo anteriormente comentado, se muestra en el cuadro 8 y figura 3:

\section{CUADRO 8. CORRELACIÓN EN LAS DIMENSIONES DE LA CS Y LT EN BANCOMER 1 Y 2}

\begin{tabular}{|l|c|c|c|}
\hline DIMENSIONES & Media CS & Media Lt & Correlación \\
\hline TANGIBILIDAD & 5,71 & 5,79 & \\
\cline { 1 - 3 } FIABILIDAD & 5,90 & 5,90 & \multirow{2}{*}{$\mathbf{0 , 8 4 9}$} \\
\cline { 1 - 3 } CAP. RESP. & 5,82 & 5,85 & \\
\cline { 1 - 3 } SEGURIDAD & 5,90 & 5,82 & \\
\cline { 1 - 3 } EMPATÍA & 6,08 & 5,92 & \\
\hline
\end{tabular}

Fuente: Elaboración propia

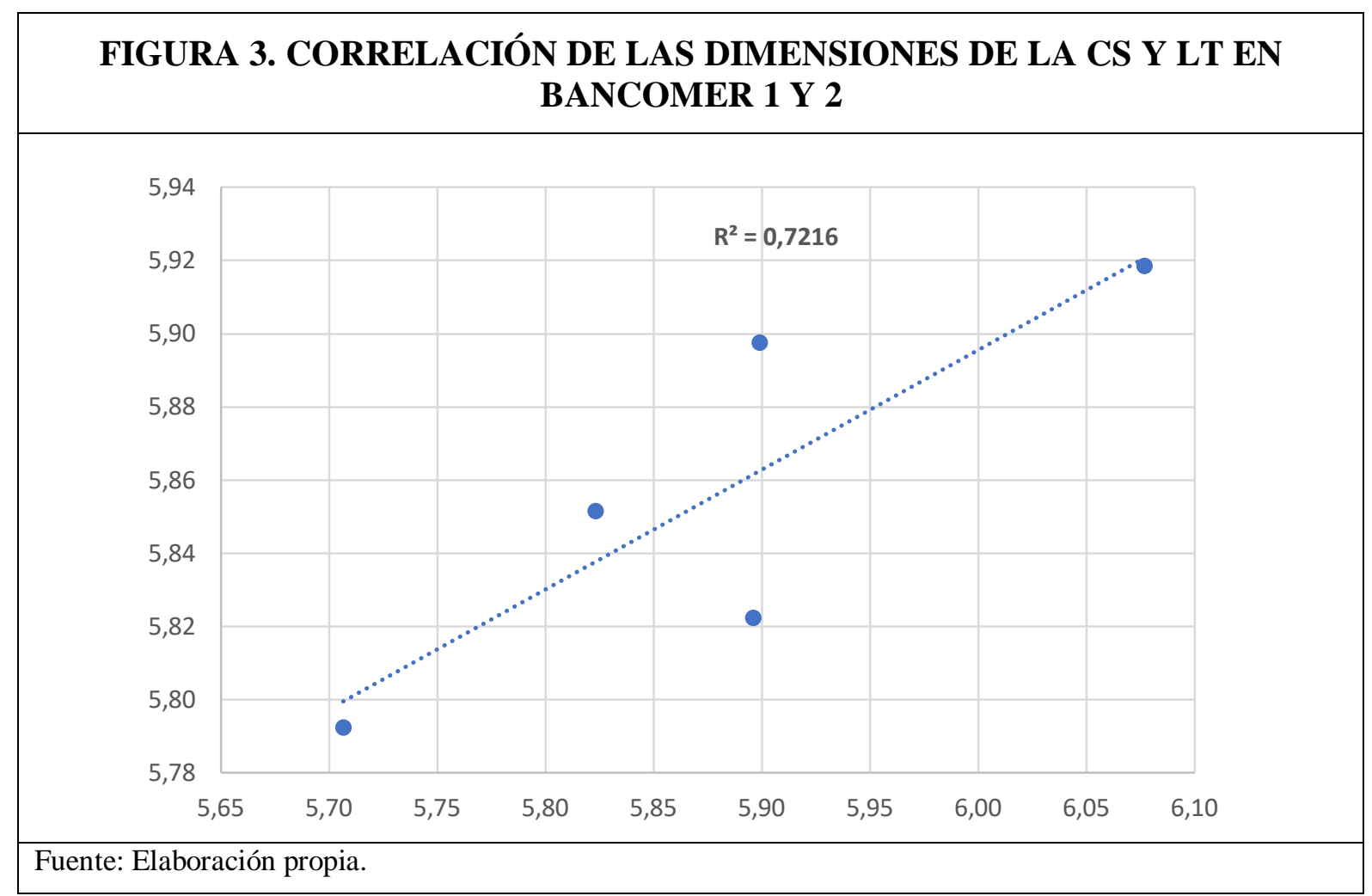


Otro apartado importante en este trabajo de investigación fue determinar como objetivo específico si existía diferencia significativa entre las medias de las dimensiones que componen a la calidad en el servicio en las dos instituciones financieras BANCOMER. Los resultados se pudieron calcular a través de un análisis de varianza (ANOVA), tal y como se muestra en el cuadro 9:

CUADRO 9. ANOVA DE LAS DIMENSIONES DE LA CS EN BANCOMER 1 Y 2

Fuente: Elaboración propia.

\begin{tabular}{|ccc|}
\hline \multicolumn{3}{|c|}{ ANÁLISIS DE VARIANZA } \\
\hline $\boldsymbol{F}$ & Probabilidad & Valor crítico para $\boldsymbol{F}$ \\
\hline 0,6380 & 0,4475 & 5,3177 \\
\hline
\end{tabular}

Los datos que muestra el cuadro 7, manifiesta que, no existe diferencia significativa entre las medias de las dimensiones de la calidad en el servicio al hacer el comparativo en las dos instituciones financieras BANCOMER en la ciudad de La Paz, México, ya que, el valor $p$ que se obtuvo durante el análisis inferencial estuvo por encima $(0,4475)$ de 0,05 (nivel de significancia); además, que el valor $f(0,6380)$ no superó el valor crítico $(5,3177)$.

\section{CONCLUSIONES}

Los resultados de este trabajo, sin duda alguna, presenta información de sumo interés tanto para la calidad del servicio como para la lealtad del cliente en el ámbito del sector bancario, los cuales, son una línea base con indicadores medibles de calidad y mejora para la industria empresarial en el sector de servicios bancarios en La Paz, México, ya que, con anterioridad, no se había presentado una investigación de estas caracaterísticas en la localidad ya mencionada.

El estudio de la calidad en el servicio y de la lealtad del cliente nunca será un tema de la mercadotecnia sencillo de abordar por parte de los empresarios, sobre todo, por el matiz subjetivo con que puede interpretarse por parte de los clientes, y es que, como mencionan Reeves y Bednard (1994), no hay una definición de calidad que sea mejor que otra, ya que, cada definición tene sus propias fortalezas y debilidades con relación a criterios en que, la medición y la generalización, la utilidad de gestión, y la pertinencia de los consumidores se presenta y adapta en su particular entorno.

Esta investigación permite concluir que BANCOMER, en la ciudad de La Paz, México, el fenómeno de la calidad en el servicio se encuentra muy bien percibida por parte de sus consumidores, ya que, al momento de hacer la evaluación descriptiva es posible aseverar por medio del análisis de medias que la CS tuvo una apreciación general de 5,88, lo que representa un juicio de valor percibido del 84 por cien; mientras que, la Lt obtuvo una media entre sus dimensiones de 5,86, lo que representó una efectividad del 83 por cien. Lo anterior, permitió evaluar de forma completa el objetivo general de este artículo que decía conocer el grado de impacto de la calidad en el servicio en la lealtad del cliente en las dos instituciones financieras $B B V A B A N C O M E R$, y es que, a través de un coeficiente de correlación de Pearson se obtuvo un valor general de 0,85 y un $\mathrm{R}^{2}$ equivalente a 0,72 ; tales resultados, indican que existe una fuerte incidencia entre la variable independiente (CS) sobre la variabe dependiente (Lt) en la investigación. 
En relación al análisis inferencial de las percepciones generadas por los clientes asiduos a BANCOMER, se pudo observar que en el segundo objetivo que citaba acerca de conocer si existía diferencia significativa entre las medias de las dimensiones de la CS, a través de un análisis de varianza (ANOVA), fue posible afirmar que no se presentó diferencia significativa, ya que el valor $p(0,4475)$ de la prueba entregó un resultado superior al valor de significancia del estudio $(0,05)$, y el valor $f(0,6380)$ fue inferior al valor crítico de $f(5,3177)$. Los resultados de cada una de las dimensiones, indican que la eficiencia de la CS y de la Lt del cliente se encuentran por encima del 80 por cien con relación a la percepión de los comensales. Con relación al objetivo general, fue posible demostrar una considerable correlación positiva entre la CS y la lt según la dimensión percibidas en las dos instituciones financieras BANCOMER en La Paz, México. El coeficiente $r$ por arriba de 0,7 indica que existe una fuerte correlación; principalmente, en las dimensiones “Seguridad y Empatía”, ya que sus evaluaciones globales estuvieron alrededor de 5,90 a 6,08. Se interpreta que la CS en las dos instituciones financieras tiene una incidencia positiva sobre la Lt, debido que, al aumentar su calidad, la lealtad hacia el cnsumo se incrementa linealmente.

\section{BIBLIOGRAFÍA}

Aldlaigan, A. H., \& Buttle, F. A. (2002): SYSTRA-SQ: a new measure of bank service quality, International Journal of Service Industry Management, vol. 13, $\mathrm{n}^{\circ} 4$, pp. 362-381. doi: http://dx.doi.org/10.1108/09564230210445041.

Avkiran, N. K. (1994): Developing an Instrument to Measure Customer Service Quality in Branch Banking. International Journal of Bank Marketing, vol. 12, n ${ }^{\circ}$ 6, pp. 10 - 18. doi:https://doi.org/10.1108/02652329410063223.

Bahia, K., \& Nantel, J. (2000): A reliable and valid measurement scale for the perceived service quality of Banks, International Journal of Bank Marketing, vol. 18, $\mathrm{n}^{0}$ 2, pp. 84-91. doi:https://doi.org/10.1108/02652320010322994

Barlow, R. G. (1992): Relationship marketing - the ultimate in customer services. Retail Control, vol. 60, n 3, pp. 29 - 37.

Bender, E. (1976): An Early Nineteenth Century Study of the Jains, Journal of the American oriental Society, vol. 96, no 1, pp. 114 - 126. doi:http://dx.doi.org/10.2307/599898

Berry, L. (1995): Relationship Marketing of Services-Growing Interest, Emerging Perspectives, Journal of the Academy of Marketing Science, vol. 23, n ${ }^{\circ}$ 4, pp. 236 - 245. doi:https://doi.org/10.1177/009207039502300402

Bitner, M. J. (1990): Evaluating Service Encounters: The Effects of Physical Surroundings and Employee Responses, Journal of Marketing, $\mathrm{n}^{\circ}$ 54, pp. 69 - 82. doi:http://dx.doi.org/10.2307/1251871

Blanchard, R. F., \& Galloway, R. L. (1994): Quality in Retail Banking, International Journal of Service Industry Management, vol. 5, $\mathrm{n}^{\mathrm{o}} \quad 4, \quad \mathrm{pp} . \quad 5 \quad-23$. doi:https://doi.org/10.1108/09564239410068670

Bolton, R. N., \& Drew, J. H. (1994): Linking Customer Satisfaction to Service Operations and Outcomes. SAGE Publications, Thousands Oaks.

Bonnet, D. G. (2002): Sample size requirements for testing and estimating coefficient alpha, Journal of Education and Behavioral Statistics, vol. 27, $\mathrm{n}^{0}$ 335, pp. 335-340. doi:10.3102/10769986027004335

Bravo, M., Vásquez, A. Z., \& Zamora, J. (2005). Loyalty in the air: Real and ficticious factors in the formation of loyalty of airline passengers, Studies and Perspectives in Tourism, vol. 14, no 2, pp. 101 - 126.

Chinomona, R., Mahlangu, D., \& Poe, D. (2013): Brand Service Quality, Satisfaction, Trust 
and Preference as Predictors of Consumer Brand Loyalty in the Retailing Industry, Mediterranean Journal of Social Sciences, vol. 4, $\mathrm{n}^{\circ}$ 14, pp. 181. Obtenido de http://www.mcser.org/journal/index.php/mjss/article/view/1591

Cobra, M. (2002): Marketing de Servicios: Estrategias para el turismo, finanzas, salud y comunicación (2e ed.), McGraw - Hill, Bogota. Obtenido de http://umecit.metabiblioteca.org/cgi-bin/koha/opac-detail.pl?biblionumber=40

Cronin, J. J., \& Taylor, S. A. (1994): SERVPERF vs SERVQUAL: Reconciling performance - based and perceptions - minus - expectations measurement of service quality, Journal of Marketing, vol. 58, $\mathrm{n}^{\mathrm{o}}$ 1, pp. 125 - 131. doi:http://dx.doi.org/10.2307/1252256

Cronin, J., \& Taylor, S. (1992): Measuring Service Quality: A reexamination and extensión, Journal of Marketing, vol. 56, nº 3, pp. 55 - 68. doi:http://doi.org/10.2307/1252296

Dabholkar, P. A., Thorpe, D. I., \& Rentz, J. O. (1996): A Measure of Service Quality for Retail Stores: Scale Development and Validation, Journal of the Academy of Marketing Science, n 24 , pp. 3 - 16. doi:https://doi.org/10.1007/BF02893933

Dávila Nima, J. (2007): Lealtad del cliente: generando y consolidandovínculos, RevistaCompetitividad, vol. 7, $\mathrm{n}^{\mathrm{o}}$ 10, pp. 52-55.

Dávila, J. A., \& Florez, M. (2008): Calidad del servicio percibida por clientes de entidades bancarias de Castilla y León y su repercusión en la satisfacción y lealtad de la misma. PECVNIA, Revista de la Facultad de Ciencias Económicas y Empresariales de la Universidad de León (en línea), $\mathrm{n}^{0}$ 7, pp. 105-128.

Dick, A. S., \& Basu, K. (1994): Customer loyalty: Toward an integrated conceptual framework, Journal of the Academy of Marketing Science, $\mathrm{n}^{\circ}$ 22, pp. 99-113.

Domínguez, S. A., \& Merino, C. (2015): ¿Por qué es importante reportar los intervalos de confianza del coeficiente alfa de Cronbach?, Revista Latinoamericana de Ciencias Sociales, Niñez y Juventud, vol. 13, n 2, pp. 1326-1328.

Duque Oliva, E., \& Canas Baquero, J. (2014): Validación del modelo SERVPERF en el ámbito, Suma de Negocios, vol. 5, $\mathrm{n}^{\circ}$ 12, $\quad$ pp. 115 - 123. doi:http://dx.doi.org/10.1016/j.neucir.2013.12.001

Ehrenberg, A., \& Goodhardt, G. (2002): Double Jeopardy Revisited, Again, Marketing Research, vol. $14, \mathrm{n}^{\circ} 1$, pp. 40-42.

Ennew, C. T., Reed, G. V., \& Binks, M. R. (1993): Importance-performance analysis and the measurement of service quality, European Journal of Marketing, vol. 27, n 2, pp .59 - 70. doi:10.1108/03090569310026402

Ehigie, B. O. (2006): Correlates of customer loyalty to their bank: a case study in Nigeria, International Journal of Bank Marketing, vol. 24, $\mathrm{n}^{\mathrm{0}} \quad 7, \quad$ pp. 494-508. doi:10.1108/02652320610712102.

González Álvarez, R. (2015): Evaluación de la calidad del servicio percibida en entidades bancarias a través de la escala SERVQUAL, Ciencia e Ingeniería Neogranadina, vol. 25, n 1 , pp. 113 - 135. doi:https://doi.org/10.18359/rcin.439

González, Z. (2001): El reto de la calidad del servicio financiero en la Comunidad Autónoma de Canarias. Ed. Fundación FYDE - Caja Canarias, Santa Cruz de Tenerife.

Grönroos, C. (1984): A service Quality model and its marketing implications. European Journal of Marketing, vol. 18, n 4, pp. 36 - 44.

Gutiérrez, H., Gutiérrez, P., Garibay, C., \& Díaz, L. (2014): Análisis multivariado y QFD como herramientas para escuchar la voz del cliente y mejorar la calidad del servicio, Ingeniare. Revista chilena de ingeniería, vol. 22, $\mathrm{n}^{\mathrm{o}} 1$, pp. 62 - 73. doi: https://dx.doi.org/10.4067/S0718-33052014000100007

Guzmán López, A., \& Cárcamo Solís, M. (2014): La evaluación de la calidad en el servicio: caso de estudio "Restaurant Familiar Los Fresnos", Acta Universitaria, vol. 24, n 3, pp. 35 - 49. doi:10.15174/au.2014.503 
Hallowell, R. (1996): The relationships of customer satisfaction, customer loyalty, and profitability: an empirical study, International Journal of Service Industry Management, vol. 7, no 4, pp. 27 - 42. doi:https://doi.org/10.1108/09564239610129931

Hayes, A. F. (2013): Introduction to mediation, moderation and conditional process analysis, The Journal of Marketing, vol. 46, n 2, pp. 60 - 72.

Hemmasi, M., Strong, K. C., \& Taylor, S. A. (1994): Measuring Service Quality for Strategic Planning and Analysis in Service Firms, Journal of Applied Business Research, vol. 10, $\mathrm{n}^{\mathrm{o}}$ 4, pp. 24 - 34.

Hernández, A., Espejo, B., González, V., \& Gómez, B. (2013): Escalas de respuesta tipo Likert: ¿Es relevante la alternativa "indiferente"?, Metodología de Encuestas, vol. 3, n 2, pp. 135 150 .

Hernández, R., Fernández, C., \& Baptista, L. (2014): Metodología de la Investigación, McGraw-Hill Education, México.

Heung, V., Wong, M. Y., \& Qu, H. (2000): Airport-restaurant service quality in Hong Kong: An application of SERVQUAL, Cornell Hospitalitu Quarterly, vol. 41, nº 3, pp .86 - 96. doi: 10.1177/001088040004100320

Jain, S. K., \& Gupta, G. (2004): Measuring Service Quality: Servqual vs. Servperf Scales, Vikalpa, vol. 29, no 2, pp. 25 - 38. doi:https://doi.org/10.1177/0256090920040203

Jensen, J. M., \& Hansen, T. (2006): An empirical examination of brand loyalty, Journal of Product \& Brand Management, vol. 15, $\mathrm{n}^{\circ}$ 7, pp. 442-449. doi:https://doi.org/10.1108/10610420610712829

Johnston, R. (1997): Identifying the critical determinants of service quality in retail banking: importance and effect, International Journal of Bank Marketing, vol. 15, nº 4, pp. 111 - 116. doi:https://doi.org/10.1108/02652329710189366

Joseph, M., McClure, C., \& Joseph, B. (1999): Service quality in the banking sector: the impact of technology on service delivery, International Journal of Bank Marketing, vol. 17, $\mathrm{n}^{\circ} 4$, pp. 182 - 193. doi:https://doi.org/10.1108/02652329910278879

Karatepe, O., Yavas, U., \& Babakus, E. (2005): Measuring service quality of banks: Scale development and validation, Journal of Retailing and Consumer Services, vol. 12, $\mathrm{n}^{\circ}$ 5, pp. 373-383. doi:10.1016/j.jretconser.2005.01.001

Kumar, V., \& Shah, D. (2004): Building and sustaining profitable customer loyalty for the 21st century. Journal of Retailing, vol. 80, $\mathrm{n}^{\circ}$ 4, pp. 317-329.

Ladhari, R. (2008): Alternative measures of service quality: a review, Managing Service Quality: An International Journal, vol. $18, \mathrm{n}^{\circ} \quad 1, \quad$ pp. $65 \quad-86$. doi:https://doi.org/10.1108/09604520810842849

Lovelock, C. H., Carrion, M. A., Reynoso, J., \& D’Andrea, G. (2004): Administración de servicios. Pearson Education, México.

Mersha, T., \& Adlakha, V. (1992): Attributes of service quality: the consumers' perspective. International Journal of Service Industry Management, vol. 3, $\mathrm{n}^{\mathrm{o}}$ 3, pp. 34 - 45. doi:. http://dx.doi.org/10.1108/09564239210015157

Montgomery, D. (2010): Control Estadístico de la Calidad, Limusa - Wiley, México D. F.

Oliver, R. (1997): Satisfaction: A behavioral perspective on the consumer, Irwin - McGrawHill, New York.

Parasuraman, A., Zeithaml, V., \& Berry, L. (1985): A Conceptual Model of Service Quality and its Implication for Future Research (SERVQUAL), Journal of Marketing, $n^{\circ}$ 49, pp. 41 - 50. doi:DOI: 10.2307/1251430

Parasuraman, A., Zeithaml, V., \& Berry, L. (1988): SERVQUAL, Journal of Retailing, 64(1), $12-40$.

Pedraja, M., \& Rivera, A. (2002): La gestión de la lealtad del cliente a la organización: Un enfoque de marketing relacional, Economía Industrial, nº 348, pp. 143-153. 
Prakash, A., \& Mohanty, R. P. (2013): Understanding service quality, Production Planning \& Control, vol. 24, no 12, pp. 1050 - 1065. doi:10.1080/09537287.2011.643929

Ramezani, A., Feiz, S., \& Baharun, R. (2015): Service Quality Measurements: A Review, International Journal of Academic Research in Business and Social Sciences, vol. 5, $\mathrm{n}^{\mathrm{o}}$ 2, pp. 267 - 286. doi:10.6007/IJARBSS/v5-i2/1484

Reeves, C. A., \& Bednard, D. A. (1994): Defining Quality: Alternatives and Implications, The Academy of Management Review, vol. 19, $\mathrm{n}^{\circ}$ 3, pp. 419-445. doi:10.2307/258934

Ruiz, J. (2006): Gestión de la Calidad en la Administración Pública (4 ed. ed.), CEDDET, Ministerio de Administraciones Públicas - INAP, Madrid.

Rust, R. T., \& Oliver, R. L. (1994): Service Quality: New directions in theory and practice. SAGE Publications, Thousand Oaks.

Sangeetha, J., \& Mahalingam, S. (2011): Service quality models in banking: a review, International Journal of Islamic and Middle Eastern Finance and Management, vol. 4, $\mathrm{n}^{\mathrm{o}}$ 1, pp. 83 - 103. doi:https://doi.org/10.1108/17538391111122221

Seth, N., Deshmukh, S. G., \& Vrat, P. (2005): Service quality models: a review, International Journal of Quality \& Reliability Management, vol. 22, n ${ }^{\circ}$ 9, pp. 913 - 949. doi:https://doi.org/10.1108/02656710510625211

Shamsudin, M., Hussain, H. I., \& Salem, M. A. (2016): Impact Of Customer Relationship Management In Bank's Loyalty Program, International Journal of Philosophy and Social Sciences, vol. 1, $\mathrm{n}^{\circ}$ 2, pp. 131-140. Obtenido de http://www.ripublication.com.

Soriano, D. R. (2002): Customers' expectations factors in restaurants: The situation in Spain, International Journal of Quality \& Reliability Management, vol. 8, $\mathrm{n}^{\circ}$ 9, pp. 1055-1067. doi:https://doi.org/10.1108/02656710210438122

Sureshchandar, G., Rajendran, C., \& Kamalanabhan, T. J. (2001): Customer perceptions of service quality: A critique, Total Quality Management, vol. 12, $\mathrm{n}^{\mathrm{o}} 1$, pp. 111-124. doi: http://dx.doi.org/10.1080/09544120020010138

Taucher, E. (1997): Bioestadísticas, Colección Textos Universitarios, Santiago de Chile.

Teas, R. K. (1993): Expectations, Performance Evaluation, and Consumers' Perceptions of, Journal of Marketing, vol. 18, n ${ }^{\circ}$ 4, pp. 18 - 34.

Vargas, M., \& Aldana, L. (2014): Calidad y servicio Conceptos y herramientas (Tercera ed.), Ecoe Ediciones, Bogota.

Zeithaml, V. (2000): Service quality, profitability, and the economic worth of customers, Journal of the Academy of Marketing Science, pp. 67 - 85. doi:http://doi.org/d9grv4.

Zeithaml, V., \& Bitner, M. (2000): Services Marketing: Integrating Customer Focus across the Firm (2nd ed.), McGraw-Hill, Boston. 